\title{
VHH-Based Bispecific Antibodies Targeting Cytokine Production
}

\author{
Maxim A. Nosenko,,2, Kamar-Sulu N. Atretkhany 1,2, Vladislav V. Mokhonov ${ }^{1,3}$, \\ Grigory A. Efimov' ${ }^{2,4}$, Andrey A. Kruglov ${ }^{2,3,5}$, Sergei V. Tillib ${ }^{2,6}$, Marina S. Drutskaya ${ }^{1,2}$ and \\ Sergei A. Nedospasov 1,2,3,5*
}

\begin{abstract}
'Engelhardt Institute of Molecular Biology, Russian Academy of Sciences, Moscow, Russia, ${ }^{2}$ Lomonosov Moscow State University, Moscow, Russia, ${ }^{3}$ Lobachevsky State University of Nizhny Novgorod, Nizhny Novgorod, Russia, ${ }^{4}$ National Research Center for Hematology, Moscow, Russia, ${ }^{5}$ German Rheumatism Research Center, Leibniz Institute, Berlin, Germany, ${ }^{6}$ Institute of Gene Biology, Russian Academy of Sciences, Moscow, Russia
\end{abstract}

Proinflammatory cytokines, such as TNF, IL-6, and IL-1, play pathogenic roles in multiple diseases and are attractive targets for biologic drugs. Because proinflammatory cytokines possess non-redundant protective and immunoregulatory functions, their systemic neutralization carries the potential for unwanted side effects. Therefore, next-generation anti-cytokine therapies would seek to selectively neutralize pathogenic cytokine signaling, leaving normal function intact. Fortunately, the biology of proinflammatory cytokines provides several such opportunities. Here, we discuss various applications of bispecific antibodies targeting cytokines with specific focus on selective TNF neutralization targeted directly to the surface of specific populations of monocytes and macrophages. These bispecific antibodies combine an anti-TNF $V_{H} H$ with $V_{H} H s$ or scFvs directed against abundant surface molecules on myeloid cells and serve to limit the bioavailability of TNF produced by these cells. Such reagents may become prototypes of a novel class of anti-cytokine biologics.

Keywords: TNF, IL-6, macrophages, single-chain antibodies, $\mathrm{V}_{\mathrm{H}} \mathrm{H}$

Many currently used therapeutic antibodies represent antagonists or inhibitors of signaling cascades that are known to be pathogenic in a particular disease state. Examples include anti-cytokine therapies and immune checkpoint inhibitors, both of which have resulted in major advances in the treatment of autoimmune diseases and cancer. One common problem with many of such inhibitors, when applied systemically, is incomplete discrimination of "pathogenic" signaling from "physiological" signaling, the latter being beneficial for the patient. Therefore, most current therapies have unwanted side effects resulting from collateral damage to beneficial or protective signaling cascades. This problem can be potentially addressed through additional specificity conferred by more sophisticated inhibitory antibodies that target their cognate antigens only in a particular organ or cell lineage.

Therapeutic bispecific antibodies have showed efficacy in both experimental animal models and in clinical trials (1), finding applications in cancer immunotherapy (2) as well as in treatment of autoimmune diseases (3) and hemophilia (4). Examples include: (i) bispecific T-cell engagers (5) that redirect the activity of $\mathrm{CD} 3+$ cytotoxic $\mathrm{T}$ lymphocytes against $\mathrm{CD} 19+$ leukemias and lymphomas (6) and EpCAM+ solid tumors (7); (ii) bispecific NK-cell engagers that redirect the activity of CD16+ natural killer cells against CEA+ solid tumors (8); (iii) bispecific molecules composed of a CD19-binding moiety and an anti-CD47 immune checkpoint inhibitor, allowing for selective CD47 blockade on malignant B cells (9); (iv) bispecific molecules composed of an a $\beta$-secretase (BACE-1)-inhibiting moiety and an anti-transferrin receptor "trojan" moiety to facilitate permeation 
of the blood-brain barrier (10); and (v) bispecific molecules composed of an anti-HIV gp41 glycoprotein moiety and an anti-CD89 moiety, designed to facilitate virus clearance by blood neutrophils (11). Several designs of bispecific antibodies have been employed, including chemically conjugated monoclonal antibodies, quadroma-produced antibodies, or genetically fused recombinant single-chain Fvs (12). The lattermost molecules lack the $\mathrm{Fc}$ region and thus have very short serum half-lives. Recently, an interesting solution to the problem of rapid clearance of these molecules was proposed in the form of RNA delivery (13). Overall, more than a dozen bispecific antibodies have now been evaluated in clinical trials.

Several bispecific antibodies targeting cytokines have been described (14), allowing for dual cytokine blockade (15-18) as well as targeted cytokine neutralization on cytokine-producing cells (19) or at particular anatomical sites, such as inflamed joints (20). One important target in anti-cytokine therapy of autoimmune diseases is TNF, and many systemic anti-TNF biologics are approved for clinical use. There are several experimental reagents that have added a second specificity to an existing antiTNF moiety. For example, a bispecific antibody directed against TNF and IL-17A was shown to be effective for the treatment of psoriasis (21). A TNF inhibitor with additional specificity to ROS-modified collagen allowed for targeted TNF inhibition in arthritic joints (20). Coppieters et al. (22) reported a highly efficient bispecific antibody that was able to bind TNF as well as an abundant serum protein (albumin), thus resulting in a significant increase of the antibody's half-life in vivo. Two different inhibitors of TNFRI signaling, each with a second specificity to serum albumin for half-life extension in vivo, are effective in mouse models of Crohn's disease and arthritis (23-25). Other studies have achieved longer half-lives and increased potencies of anti-TNF inhibitors by various types of dimerization or oligomerization (26-28) allowing the demonstration of their biological activity in mouse disease models. Although this was not directly determined, it may be assumed that all of these TNF inhibitors, including bispecifics, neutralized TNF produced by multiple cellular sources in a systemic fashion.

In our studies employing conditional gene targeting, we found that TNF produced by myeloid cells is pathogenic in several experimental mouse disease models (29-32). Assuming that TNF from other immune and non-immune sources may possess beneficial functions (33-35), we wanted to design an approach to pharmacologically limit TNF production only by myeloid cells. To this end, we designed, produced, and evaluated bispecific antibodies that bind TNF with one arm and engage surface molecules abundantly expressed on myeloid cells through another arm. Two such potential surface markers-F4/80 (EMR1, the product of the Adgre1 gene) and CD11b (Mac-1a, Integrin $\alpha \mathrm{M}$, the product of the Itgam gene; expressed by myeloid cells, NK, and some other cells) can be employed.

The discovery of heavy-chain-only antibodies in Camelidae (36) led to the development of new technologies based on the ability to generate modular, high affinity binders $\left(\mathrm{V}_{\mathrm{H}} \mathrm{Hs}\right)$ specific to almost any protein. One particular aspect that drew our attention was the usefulness of $\mathrm{V}_{\mathrm{H}} \mathrm{Hs}$ in creating bispecific reagents, as two or even three $\mathrm{V}_{\mathrm{H}} \mathrm{Hs}$ can be easily combined in a single polypeptide chain by the methods of genetic engineering (19) and expressed in prokaryotic systems. In order to specifically target TNF produced by myeloid cells, we have initially utilized a singlechain antibody to murine F4/80, which is exclusively expressed on myeloid cells with abundant expression on the surface of all mature macrophages (including microglia), Langerhans cells, and to a lesser degree on blood monocytes (37). We subsequently generated a novel $\mathrm{V}_{\mathrm{H}} \mathrm{H}$ by immunizing a Bactrian camel with recombinant murine F4/80 and genetically fused it to an antihTNF $V_{H} H(19)$. Because of the specificity of this reagent to human TNF, all subsequent in vitro and in vivo experiments were performed using human TNF knock-in mice (38). Collectively, for all these bispecific antibodies, a term myeloid cell-specific TNF inhibitor (MYSTI) has been coined. Figure 1 outlines the design, purification, and experimental protocols for evaluation of these anti-TNF bispecific $\mathrm{V}_{\mathrm{H}} \mathrm{H}$-based reagents.

Using flow cytometry, we found that MYSTI (exemplified here by MYSTI-2) binds to the surface of murine macrophages, competes with another anti-F4/80 reagent for this binding (Figures 2A,B), and attracts exogenously added human TNF to the surface of macrophages (Figure $2 \mathrm{C}$ ). We then performed experiments to prove that endogenously produced TNF can also be retained on the cell surface. To this end, bone marrow-derived macrophages from humanized TNF knock-in (hTNF KI) mice(38) were incubated with MYSTI-2, or with control TNF-neutralizing antibodies lacking anti-F4/80 targeting module (referred here as systemic TNF inhibitor or STI), then washed and activated with LPS. As shown in Figure 2D, the amount of biologically active hTNF released into culture medium is significantly lower in the presence of MYSTI as compared to STI, suggesting that MYSTI indeed retained hTNF on the surface of macrophages and may limit its systemic release in vivo.

To get a better insight into the fate of hTNF and of MYSTI after its binding to the surface of macrophage, we utilized confocal microscopy, as outlined in Figures 1C-F. As expected, FITC-labeled MYSTI could stain these cells and was detected on the surface of activated macrophages as early as 15 min following incubation and-interestingly - up to $18 \mathrm{~h}$ later although in diminished amounts, consistent with our previous results (Figure 2E, top row and data not shown). In contrast, STI briefly stained activated macrophages after $15 \mathrm{~min}$ of incubation, while upon subsequent washing, such staining rapidly disappeared (Figure 2E, bottom row and data not shown). Since we did not detect binding of STI to unstimulated macrophages (data not shown), we hypothesized that such staining is due to recognition of transmembrane TNF (tmTNF) on the surface of activated macrophages. MYSTI was able to bind and retain human TNF produced by macrophages from hTNF KI mice (as indicated by the arrows in Figure 2E, top row) and exogenously added human TNF (Figure. 2F). We also detected rapid internalization of MYSTI (Figure 2F) starting from approximately $30 \mathrm{~min}$ of incubation with macrophages. Both unbound (Figure 2F, left) and TNF-bound (Figure 2F, right) bispecific antibodies were internalized, suggesting that internalization does not require TNF recognition by MYSTI. Exogenously added TNF, labeled by a secondary PE-conjugated antibody, could be detected on the surface of macrophages for at least 1-2 $\mathrm{h}$ (Figure 2F and data not shown). 


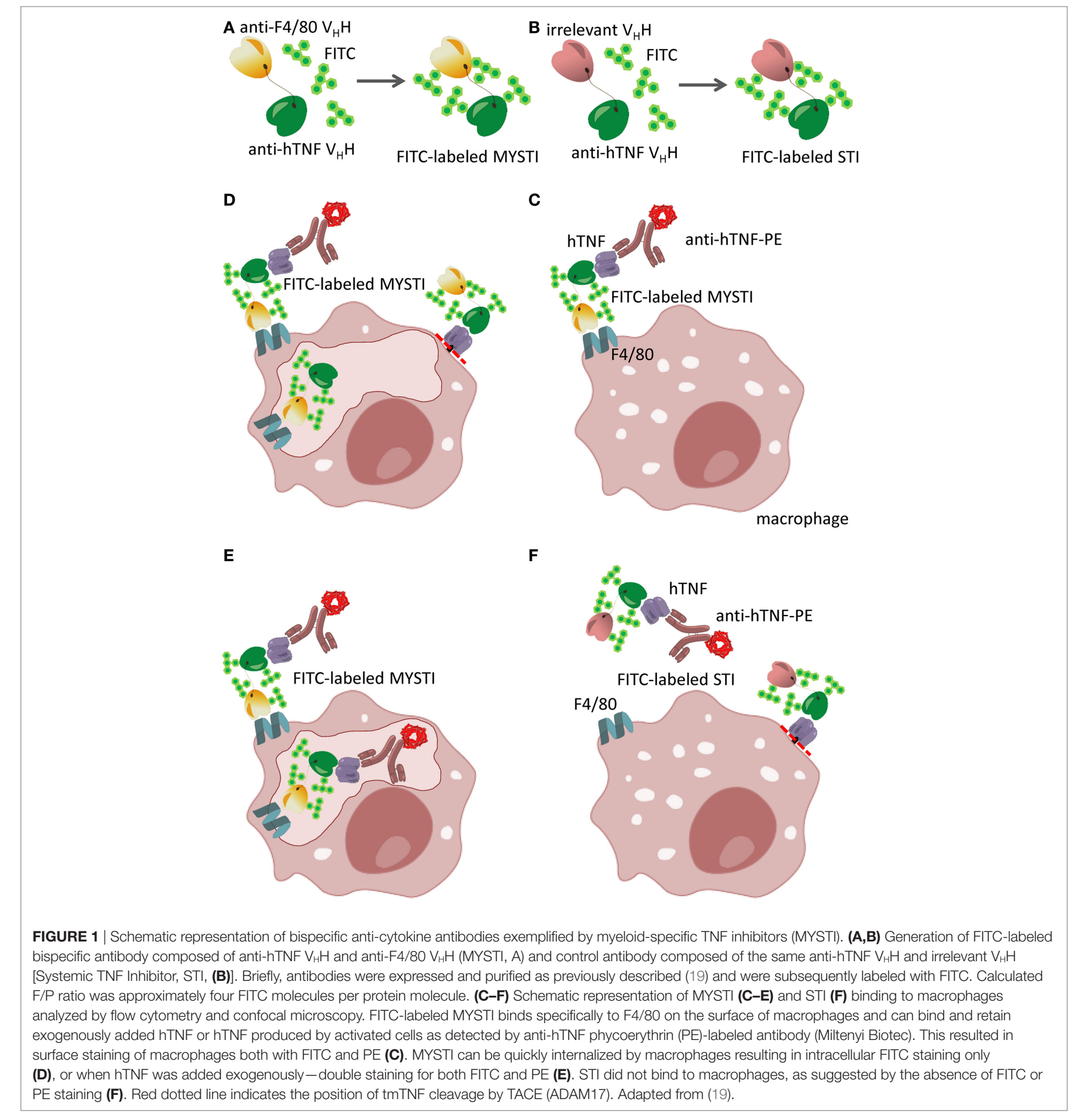

Based on the encouraging finding that MYSTI, an antibody with two $\mathrm{V}_{\mathrm{H}} \mathrm{H}$ domains, may be sufficiently long-lived on the surface of cytokine-producing cells, we evaluated these reagents in vivo. In LPS/D-Gal lethal toxicity model, pathogenic TNF is known to be produced by myeloid cells (39) and animals become moribund within 6-8 h (19). In this model, administration of MYSTI at $3 \mathrm{mg} / \mathrm{kg}$ completely protected mice, while the same dose of the control reagents (such as STI that contained exactly the same TNF-binding and neutralizing $\mathrm{V}_{\mathrm{H}} \mathrm{H}$ module) failed to do so (19). Moreover, the results suggest that MYSTI retained its protective ability even at $1-1.5 \mathrm{mg} / \mathrm{kg}$ dose and modification with FITC did not affect its properties (Figure 2G and data not shown), thus allowing us to further investigate its fate in vivo. As an additional control, we used Infliximab as a systemic TNF inhibitor control, which also protected mice against LPS/DGal-induced hepatotoxicity at the dose of $1.5 \mathrm{mg} / \mathrm{kg}$ (data not shown); however, differences in molecular weight and avidity should be taken in account when comparing full-length systemic 


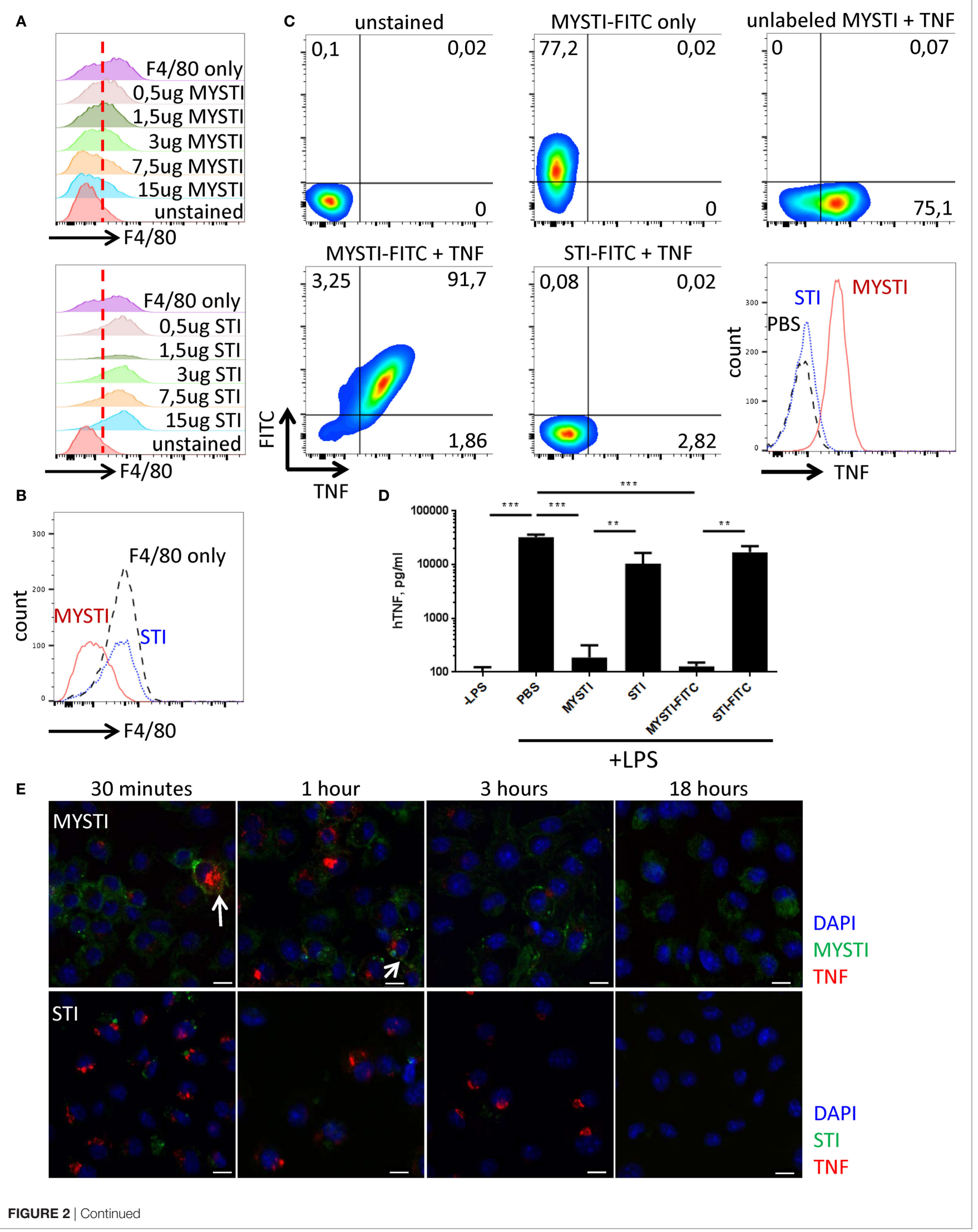



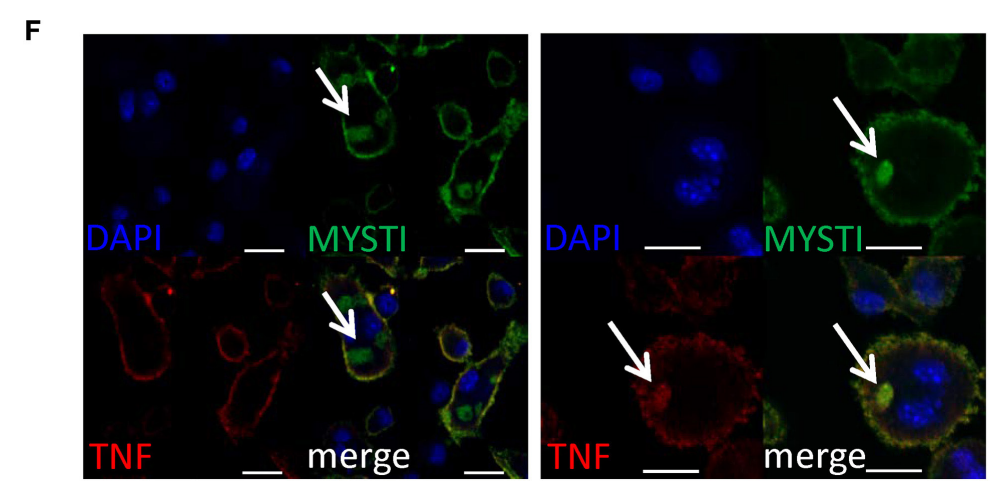

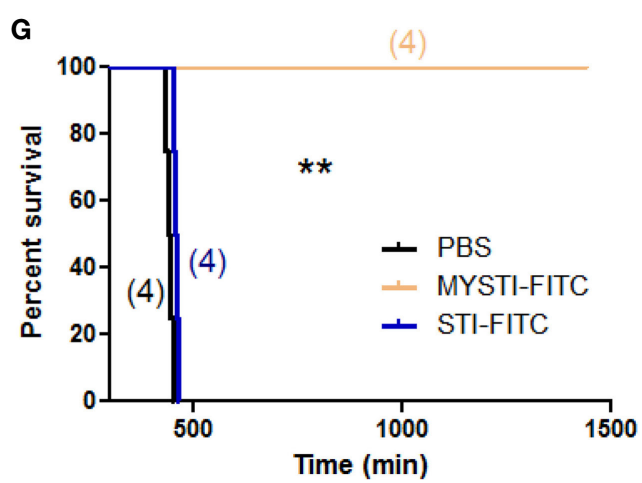

FIGURE 2 | Characterization of MYSTI and STI interaction with macrophages in vitro and in vivo. (A) MYSTI, but not STI, competes with anti-F4/80 antibody for binding sites resulting in reduced staining for F4/80. Staining of macrophages with anti-F4/80 antibody in the presence of indicated concentrations of MYSTI (top panel) or STI (bottom panel). Red dotted line splits F4/80- and F4/80+ cells on the left and on the right, respectively. Briefly, bone marrow-derived macrophages were simultaneously incubated with anti-F4/80 antibody (clone BM8 that competed for binding to F4/80 with anti F4/80 $\mathrm{V}_{\mathrm{H}} \mathrm{H}$, used in MYSTI) and indicated amounts of MYSTI or STI. All cells were gated as ViabilityDye-CD11b+. (B) Staining of macrophages with anti-F4/80 only or in the presence of MYSTI or STI. Data indicate that MYSTI selectively binds to F4/80. (C) MYSTI, but not STI, binds to the surface of macrophages and retains exogenously added hTNF. Surface staining of macrophages with MYSTI or STI and hTNF. Top row represents unstained or single stained cells as controls. Bottom row represents staining of macrophages with MYSTI-FITC and hTNF (left), STI-FITC and hTNF (middle), and a summarizing histogram of hTNF staining (right). Briefly, bone marrow-derived macrophages were subsequently incubated with MYSTI or STI followed by recombinant human TNF and with anti-hTNF antibody incubations. All cells were gated as VD-CD11 b+. (D) MYSTI, but not STI, prevents hTNF release into the culture medium by LPS-stimulated macrophages. BMDM from hTNFKI mice were cultured with MYSTI or STI antibodies or PBS, washed once, and stimulated with $100 \mathrm{ng} / \mathrm{ml}$ of LPS from E. coli. Release of hTNF into culture medium was measured $4 \mathrm{~h}$ following induction with LPS using Ready-Set-Go ELISA kit (eBioscience). ${ }^{\star *} p<0.01$; ${ }^{\star * *} p<0.001$ in one-way ANOVA. (E) Dynamics of MYSTI and STI staining on LPS-activated macrophages as revealed by confocal microscopy. Briefly, macrophages were activated with $100 \mathrm{ng} / \mathrm{ml}$ of LPS for $3 \mathrm{~h}$, followed by incubation with FITC-labeled MYSTI or STI for 15 min, then washed, and fixed at indicated time points. Fixed cells were consequently permeabilized and stained with anti-hTNF Ab labeled with PE. Starting from 30 min of incubation, MYSTI could be detected both on macrophage surface and inside the cells, while weak binding of STI was observed only after 30 min of incubation. Arrows show co-staining of MYSTI and anti-hTNF. Scale bars - $10 \mu m$. (F) MYSTI is internalized by macrophages. Confocal microscope images of macrophages stained with MYSTI (green), anti-hTNF (red), and counterstained with DAPI (blue). Briefly, cells were consequently incubated with MYSTI-FITC, recombinant hTNF, and anti-hTNF labeled with PE and then fixed. On each of the two images, top left part represents DAPI staining, top rightMYSTI-FITC, bottom left-anti-hTNF-PE, and bottom right-merged picture. Arrows show internalized MYSTI bound (right image) or not bound to hTNF (left image). Scale bars $-20 \mu \mathrm{m}$. (G) FITC-labeled MYSTI retains its ability to protect mice in the model of LPS/D-Gal-induced hepatotoxicity. Briefly, mice were injected i.p. with $1.5 \mathrm{mg} / \mathrm{kg}$, STI, or PBS and after 30 min were injected with lethal dose of LPS/D-Gal.

TNF-inhibitors with MYSTI. Additionally, MYSTI was active in anti-collagen antibody transfer arthritis model (data not shown). Another potential target for the "second specificity" is CD11b for which a $\mathrm{V}_{\mathrm{H}} \mathrm{H}$ was recently reported (40). However, expression of this molecule is not strictly restricted to myeloid cells $(41,42)$ and, additionally, F4/80 appears to be expressed at significantly higher levels, as compared to CD11b [according to mass spectrometric database (43)].

\section{CONCLUDING REMARKS AND FUTURE PERSPECTIVES}

The remarkable success of anti-cytokine therapy in treating autoimmune and other diseases suggests that bispecific antibodies targeting pro-inflammatory cytokines, such as TNF or IL-6, will be developed and used. $\mathrm{V}_{\mathrm{H}} \mathrm{H}$ technology has provided attractive antigen-binding modules for such bifunctional antibodies that simplify their engineering, expression, and purification. The central issue here is the nature of the "second specificity." These may include additional anti-cytokine moieties or binding modules directing these reagents to either specific organs or cell types. Our own studies explored the possibility of targeting anticytokine antibodies to the surface of specific TNF-producing cell types, as we believe that some cells represent predominantly pathogenic sources of cytokine, at least in a particular disease or disease state. We continue to evaluate the features of selective TNF inhibitors with a focus on their in vivo ability to bind and neutralize TNF produced by myeloid cells, but not by other cell types. We aim to expand this concept to other pro-inflammatory cytokines, such as IL-6, using $\mathrm{V}_{\mathrm{H}} \mathrm{Hs}$ generated against human IL-6 (44), although the safety of myeloid-specific IL-6 inhibitors needs to be assessed with regards to IL-6's role in the development of lymphocytes (45). This approach is a pharmacological analog of inducible cell type-restricted gene ablation in vivo, with the advantage that the effects of antibodies are reversible and more relevant for preclinical evaluation. Although ongoing studies are mostly performed in animal models, one may expect that some of these $\mathrm{V}_{\mathrm{H}} \mathrm{H}$-based multispecific biologics will be eventually approved for human therapy, as has already happened for several such reagents utilizing more conventional antigenbinding modules, such as scFv.

\section{ETHICS STATEMENT}

All manipulations with animals were carried out in accordance with recommendations in the Guide for the Care and Use of Laboratory Animals (NRC 2011), the European Convention for the Protection of Vertebrate Animals Used for Experimental and 
Other Scientific Purposes, Council of Europe (ETS 123), and "The Guidelines for Manipulations with Experimental Animals" (the decree of the Presidium of the Russian Academy of Sciences of April 02, 1980, no. 12000-496). All animal procedures were approved by Scientific Council of the Engelhardt Institute of Molecular Biology.

\section{AUTHOR CONTRIBUTIONS}

$\mathrm{MN}, \mathrm{K}-\mathrm{SA}, \mathrm{GE}, \mathrm{AK}, \mathrm{MD}$, and $\mathrm{SN}$ designed the research and analyzed the data; MN, K-SA, and MD performed the experiments; VM, GE, and ST developed and produced bispecific reagents; all authors contributed to writing the manuscript.

\section{REFERENCES}

1. Kontermann RE, Brinkmann U. Bispecific antibodies. Drug Discov Today (2015) 20:838-47. doi:10.1016/j.drudis.2015.02.008

2. Chen S, Li J, Li Q, Wang Z. Bispecific antibodies in cancer immunotherapy. Hum Vaccin Immunother (2016) 12:2491-500. doi:10.1080/21645515.2016.1 187802

3. Baker KF, Isaacs JD. Novel therapies for immune-mediated inflammatory diseases: what can we learn from their use in rheumatoid arthritis, spondyloarthritis, systemic lupus erythematosus, psoriasis, Crohn's disease and ulcerative colitis? Ann Rheum Dis (2017). doi:10.1136/annrheumdis-2017211555

4. Kitazawa T, Igawa T, Sampei Z, Muto A, Kojima T, Soeda T, et al. A bispecific antibody to factors IXa and X restores factor VIII hemostatic activity in a hemophilia A model. Nat Med (2012) 18:1570-4. doi:10.1038/nm.2942

5. Zhang X, Yang Y, Fan D, Xiong D. The development of bispecific antibodies and their applications in tumor immune escape. Exp Hematol Oncol (2017) 6:12. doi:10.1186/s40164-017-0072-7

6. Wolach O, Stone RM. Blinatumomab for the treatment of Philadelphia chromosome-negative, precursor B-cell acute lymphoblastic leukemia. Clin Cancer Res (2015) 21:4262-9. doi:10.1158/1078-0432.CCR-15-0125

7. Heiss MM, Strohlein MA, Jager M, Kimmig R, Burges A, Schoberth A, et al. Immunotherapy of malignant ascites with trifunctional antibodies. Int J Cancer (2005) 117:435-43. doi:10.1002/ijc.21165

8. Li J, Zhou C, Dong B, Zhong H, Chen S, Li Q, et al. Single domain antibody-based bispecific antibody induces potent specific anti-tumor activity. Cancer Biol Ther (2016) 17:1231-9. doi:10.1080/15384047.2016. 1235659

9. Dheilly E, Moine V, Broyer L, Salgado-Pires S, Johnson Z, Papaioannou A, et al. Selective blockade of the ubiquitous checkpoint receptor CD47 is enabled by dual-targeting bispecific antibodies. Mol Ther (2017) 25:523-33. doi:10.1016/j.ymthe.2016.11.006

10. Joy Yu Y, Atwal JK, Zhang Y, Tong RK, Wildsmith KR, Tan C, et al. Therapeutic bispecific antibodies cross the blood-brain barrier in nonhuman primates. Sci Transl Med (2014) 6:261 ra154. doi:10.1126/scitranslmed.3009835

11. Yu X, Duval M, Gawron M, Posner MR, Cavacini LA. Overcoming the constraints of anti-HIV/CD89 bispecific antibodies that limit viral inhibition. J Immunol Res (2016) 2016:9425172. doi:10.1155/2016/9425172

12. Kontermann RE. Dual targeting strategies with bispecific antibodies. MAbs (2012) 4:182-97. doi:10.4161/mabs.4.2.19000

13. Stadler CR, Bahr-Mahmud H, Celik L, Hebich B, Roth AS, Roth RP, et al. Elimination of large tumors in mice by mRNA-encoded bispecific antibodies. Nat Med (2017) 23(7):815-7. doi:10.1038/nm.4356

14. Drutskaya MS, Efimov GA, Kruglov AA, Nedospasov SA. Can we design a better anti-cytokine therapy? J Leukoc Biol (2017). doi:10.1189/jlb.3MA0117$025 \mathrm{R}$

15. Torres T, Romanelli M, Chiricozzi A. A revolutionary therapeutic approach for psoriasis: bispecific biological agents. Expert Opin Investig Drugs (2016) 25(7):751-4. doi:10.1080/13543784.2016.1187130

\section{ACKNOWLEDGMENTS}

The authors would like to thank Ruslan Zvartsev for help in performing experiments. They would also like to thank both reviewers and editor for helpful comments, critiques, and suggestions.

\section{FUNDING}

This study was supported by the Russian Science Foundation grant \#14-50-00060. Experiments utilizing flow cytometry using BD FACSCantoII (Figures 2A-C) were supported by the Russian Science Foundation grant \#14-25-00160.

16. Kim Y, Yi H, Jung H, Rim YA, Park N, Kim J, et al. A dual target-directed agent against interleukin- 6 receptor and tumor necrosis factor $\alpha$ ameliorates experimental arthritis. Sci Rep (2016) 6:20150. doi:10.1038/srep20150

17. Fischer JAA, Hueber AJ, Wilson S, Galm M, Baum W, Kitson C, et al. Combined inhibition of tumor necrosis factor $\alpha$ and interleukin-17 as a therapeutic opportunity in rheumatoid arthritis: development and characterization of a novel bispecific antibody. Arthritis Rheumatol (2015) 67:51-62. doi:10.1002/ art.38896

18. Qi J, Kan F, Ye X, Guo M, Zhang Y, Ren G, et al. A bispecific antibody against IL-1 $\beta$ and IL-17A is beneficial for experimental rheumatoid arthritis. Int Immunopharmacol (2012) 14:770-8. doi:10.1016/j.intimp.2012.10.005

19. Efimov GA, Kruglov AA, Khlopchatnikova ZV, Rozov FN, Mokhonov VV, Rose-John S, et al. Cell-type-restricted anti-cytokine therapy: TNF inhibition from one pathogenic source. Proc Natl Acad Sci U S A (2016) 113:3006-11. doi:10.1073/pnas.1520175113

20. Hughes C, Faurholm B, Dell'Accio F, Manzo A, Seed M, Eltawil N, et al. Human single-chain variable fragment that specifically targets arthritic cartilage. Arthritis Rheum (2010) 62:1007-16. doi:10.1002/art.27346

21. Silacci M, Lembke W, Woods R, Attinger-Toller I, Baenziger-Tobler N, Batey S, et al. Discovery and characterization of COVA322, a clinical-stage bispecific TNF/IL-17A inhibitor for the treatment of inflammatory diseases. MAbs (2016) 8:141-9. doi:10.1080/19420862.2015.1093266

22. Coppieters K, Dreier T, Silence K, De Haard H, Lauwereys M, Casteels P, et al. Formatted anti-tumor necrosis factor $\alpha \mathrm{VHH}$ proteins derived from camelids show superior potency and targeting to inflamed joints in a murine model of collagen-induced arthritis. Arthritis Rheum (2006) 54:1856-66. doi:10.1002/ art. 21827

23. Steeland S, Puimège L, Vandenbroucke RE, Van Hauwermeiren $F$, Haustraete J, Devoogdt N, et al. Generation and characterization of small single domain antibodies inhibiting human tumor necrosis factor receptor 1 . J Biol Chem (2015) 290:4022-37. doi:10.1074/jbc.M114.617787

24. Schmidt EM, Davies M, Mistry P, Green P, Giddins G, Feldmann M, et al. Selective blockade of tumor necrosis factor receptor I inhibits proinflammatory cytokine and chemokine production in human rheumatoid arthritis synovial membrane cell cultures. Arthritis Rheum (2013) 65:2262-73. doi:10.1002/art.38055

25. McCann FE, Perocheau DP, Ruspi G, Blazek K, Davies ML, Feldmann M, et al. Selective tumor necrosis factor receptor I blockade is antiinflammatory and reveals immunoregulatory role of tumor necrosis factor receptor II in collagen-induced arthritis. Arthritis Rheumatol (2014) 66:2728-38. doi:10.1002/ art. 38755

26. Conrad U, Plagmann I, Malchow S, Sack M, Floss DM, Kruglov AA, et al. ELPylated anti-human TNF therapeutic single-domain antibodies for prevention of lethal septic shock. Plant Biotechnol J (2011) 9:22-31. doi:10.1111/j.1467-7652.2010.00523.x

27. Plagmann I, Chalaris A, Kruglov AA, Nedospasov S, Rosenstiel P, Rose-John $\mathrm{S}$, et al. Transglutaminase-catalyzed covalent multimerization of camelidae anti-human TNF single domain antibodies improves neutralizing activity. J Biotechnol (2009) 142:170-8. doi:10.1016/j.jbiotec.2009.04.002 
28. Beirnaert E, Desmyter A, Spinelli S, Lauwereys M, Aarden L, Dreier T, et al. Bivalent Llama single-domain antibody fragments against tumor necrosis factor have picomolar potencies due to intramolecular interactions. Front Immunol (2017) 8:867. doi:10.3389/fimmu.2017.00867

29. Awad AS, You H, Gao T, Cooper TK, Nedospasov SA, Vacher J, et al. Macrophage-derived tumor necrosis factor- $\alpha$ mediates diabetic renal injury. Kidney Int (2015) 88:722-33. doi:10.1038/ki.2015.162

30. Kruglov A, Tumanov A, Grivennikov SI, Shebzukhov YV, Kuchmiy AA, Efimov GA, et al. Modalities of experimental TNF blockade in vivo: mouse models. In: Wallach D, Kovalenko A, Feldmann M, editors. Advances in TNF Family Research: Proceedings of the 12th International TNF Conference, 2009. New York, NY: Springer New York (2011). p. 421-31.

31. Kruglov AA, Lampropoulou V, Fillatreau S, Nedospasov SA. Pathogenic and protective functions of TNF in neuroinflammation are defined by its expression in T lymphocytes and myeloid cells. J Immunol (2011) 187:5660-70. doi:10.4049/jimmunol.1100663

32. Atretkhany KSN, Nosenko MA, Gogoleva VS, Zvartsev RV, Qin Z, Nedospasov SA, et al. TNF neutralization results in the delay of transplantable tumor growth and reduced MDSC accumulation. Front Immunol (2016) 7:147. doi:10.3389/fimmu.2016.00147

33. Tumanov AV, Grivennikov SI, Kruglov AA, Shebzukhov YV, Koroleva EP, Piao Y, et al. Cellular source and molecular form of TNF specify its distinct functions in organization of secondary lymphoid organs. Blood (2010) 116:3456-64. doi:10.1182/blood-2009-10-249177

34. Allie N, Grivennikov SI, Keeton R, Hsu N-J, Bourigault M-L, Court N, et al. Prominent role for $\mathrm{T}$ cell-derived tumour necrosis factor for sustained control of Mycobacterium tuberculosis infection. Sci Rep (2013) 3:1809. doi:10.1038/ srep01809

35. Kroetsch JT, Levy AS, Zhang H, Aschar-Sobbi R, Lidington D, Offermanns S, et al. Constitutive smooth muscle tumour necrosis factor regulates microvascular myogenic responsiveness and systemic blood pressure. Nat Commun (2017) 8:14805. doi:10.1038/ncomms14805

36. Hamers-Casterman C, Atarhouch T, Muyldermans S, Robinson G, Hammers C, Songa EB, et al. Naturally occurring antibodies devoid of light chains. Nature (1993) 363:446-8. doi:10.1038/363446a0

37. Austyn JM, Gordon S. F4/80, a monoclonal antibody directed specifically against the mouse macrophage. Eur J Immunol (1981) 11:805-15. doi:10.1002/ eji. 1830111013

38. Olleros ML, Chavez-Galan L, Segueni N, Bourigault ML, Vesin D, Kruglov AA, et al. Control of mycobacterial infections in mice expressing human tumor necrosis factor (TNF) but not mouse TNF. Infect Immun (2015) 83:3612-23. doi:10.1128/IAI.00743-15

39. Grivennikov SI, Tumanov AV, Liepinsh DJ, Kruglov AA, Marakusha BI, Shakhov AN, et al. Distinct and nonredundant in vivo functions of TNF produced by $\mathrm{T}$ cells and macrophages/neutrophils: protective and deleterious effects. Immunity (2005) 22:93-104. doi:10.1016/j.immuni. 2004.11.016

40. Rashidian M, Keliher EJ, Bilate AM, Duarte JN, Wojtkiewicz GR, Jacobsen JT, et al. Noninvasive imaging of immune responses. Proc Natl Acad Sci U S A (2015) 112:6146-51. doi:10.1073/pnas.1502609112

41. Kawai K, Tsuno NH, Matsuhashi M, Kitayama J, Osada T, Yamada J, et al. CD11b-mediated migratory property of peripheral blood B cells. J Allergy Clin Immunol (2005) 116:192-7. doi:10.1016/j.jaci.2005.03.021

42. Christensen JE, Andreasen SO, Christensen JP, Thomsen AR. CD11b expression as a marker to distinguish between recently activated effector CD8(+) $\mathrm{T}$ cells and memory cells. Int Immunol (2001) 13:593-600. doi:10.1093/ intimm/13.4.593

43. Bausch-Fluck D, Hofmann A, Bock T, Frei AP, Cerciello F, Jacobs A, et al. A mass spectrometric-derived cell surface protein atlas. PLoS One (2015) 10:e0121314. doi:10.1371/journal.pone.0121314

44. Tillib SV, Efimov GA, Gubernatorova EO, Goryainova OS, Ivanova TI, Bocharov AA, et al. Preparation and characterization of recombinant single-chain llama antibodies that specifically bind to human interleukin-6. Russ J Immunol (2015) 9:400-9.

45. Yang R, Masters AR, Fortner KA, Champagne DP, Yanguas-Casás N, Silberger DJ, et al. IL- 6 promotes the differentiation of a subset of naive CD8+ T cells into IL-21-producing B helper CD8+ T cells. J Exp Med (2016) 213(11):2281-91. doi:10.1084/jem.20160417

Conflict of Interest Statement: GE, AK, and SN are coauthors of the patent application describing the initial version of MYSTI. Other authors declare no conflict of interest.

Copyright (c) 2017 Nosenko, Atretkhany, Mokhonov, Efimov, Kruglov, Tillib, Drutskaya and Nedospasov. This is an open-access article distributed under the terms of the Creative Commons Attribution License (CC BY). The use, distribution or reproduction in other forums is permitted, provided the original author(s) or licensor are credited and that the original publication in this journal is cited, in accordance with accepted academic practice. No use, distribution or reproduction is permitted which does not comply with these terms. 